\title{
Multiple Shooting Method for Two-Point Boundary Value Problems*
}

\author{
David D. Morrison \\ University of Texas, Austin, Texas \\ JAMES D. RILEY \\ Aerospace Corporation, Los Angeles, Calif. \\ John F. Zancanaro \\ Space Technology Laboratories, Inc., Los Angeles, Calif.
}

\section{Introduction}

The common techniques for solving two-point boundary value problems can be classified as either "shooting" or "finite difference" methods. Central to a shooting method is the ability to integrate the differential equations as an initial value problem with guesses for the unknown initial values. This ability is not required with a finite difference method, for the unknowns are considered to be the values of the true solution at a number of interior mesh points. Each method has its advantages and disadvantages. One serious shortcoming of shooting becomes apparent when, as happens altogether too often, the differential equations are so unstable that they "blow up" before the initial value problem can be completely integrated. This can occur even in the face of extremely accurate guesses for the initial values. Hence, shooting seems to offer no hope for some problems. A finite difference method does have a chance for it tends to keep a firm hold on the entire solution at once. The purpose of this note is to point out a compromising procedure which endows shooting-type methods with this particular advantage of finite difference methods. For such problems, then, all hope need not be abandoned for shooting methods. This is desirable because shooting methods are generally faster than finite difference methods.

The organization is as follows:

I. The two-point boundary value problem is stated in quite general form.

II. A particular shooting method is described which is designed to solve the problem in this form.

* Work supported by a research contract at Space Technology Laboratories, Inc.
III. The two-point boundary value problem is then restated in such a way that:

(a) the restatement still falls within the general form, and

(b) the shooting method now has a better chance of success when the equations are unstable.

\section{The Method}

I. The two-point boundary problem may be stated as follows:

$$
\dot{y}=g(y, t), \quad f\left[y\left(t_{0}\right), y(T)\right]=0
$$

where $y, g$ and $f$ are vectors of order $n ; f$ and $g$ may be nonlinear.

II. A shooting method which has been successful in many cases is the following: Let $y(t)$ be the true solution of $(1)$, and let $y_{0}=y\left(t_{0}\right)$. Let $\bar{y}_{0}$ be an approximation to $y_{0}$, and define $\bar{y}(t)$ to be the solution of the initial value problem:

$$
\dot{\bar{y}}=g(\bar{y}, t), \quad \bar{y}\left(t_{0}\right)=\bar{y}_{0} .
$$

Thus $\bar{y}$ satisfies the differential equation, but not (in general) the boundary conditions; that is,

$$
f\left[\bar{y}\left(t_{0}\right), \bar{y}(T)\right]=\epsilon .
$$

Assume the existence of the partial derivatives necessary to construct the matrices $P, M$ and $N$, where

$$
\begin{array}{r}
p_{i, j}=\frac{\partial g_{i}(t)}{\partial y_{j}(t)}, \quad m_{i, j}=\frac{\partial f_{i}}{\partial y_{j}\left(t_{0}\right)}, \quad n_{i, j}=\frac{\partial f_{i}}{\partial y_{j}(T)} \\
(i, j=1,2, \cdots, n) .
\end{array}
$$

Let $\eta(t)=y(t)-\bar{y}(t)$. Finding $y(t)$ is then equivalent to finding $\eta(t)$ so that

$$
\begin{aligned}
& \dot{\bar{y}}+\dot{\eta}=g(\bar{y}+\eta, t), \\
& f\left[\bar{y}\left(t_{0}\right)+\eta\left(t_{0}\right), \bar{y}(T)+\eta(T)\right]=0 .
\end{aligned}
$$

Expanding these expressions in a first order Taylor series and using (2) gives the approximate formula

$$
\dot{\eta}=P \eta, \quad M \eta\left(t_{0}\right)+N \eta(T)=-\epsilon .
$$

The partial derivatives are to be evaluated for the approximate solution $\bar{y}$.

This suggests the following iterative procedure:

1. Simultaneously integrate from $t_{0}$ to $T$

$$
\dot{U}=P U, \quad U\left(t_{0}\right)=U_{0}
$$


and

$$
\dot{\bar{y}}=g(\bar{y}, t), \quad \bar{y}\left(\ell_{0}\right)=\bar{y}_{0},
$$

where $U_{0}$ is the identity matrix of order $n$, except that the $i$ th diagonal element of $U_{0}$ may be suppressed if the $i$ th component of $y\left(t_{0}\right)$ is a known condition. If $k$ components of $y\left(t_{0}\right)$ are known, then only $n(n-k)$ variational equations need to be integrated instead of $n^{2}$.

2. Compute $M, N$ and $\epsilon$.

3. Solve the linear algebraic system $\left[M U_{0}+N U(T)\right] \alpha=-\epsilon$. Then $\eta=U \alpha$ satisfies the required equations (6) as may be verified by direct substitution.

4. Form $\eta_{0}=U_{0} \alpha$ which provides a correction to $\bar{y}\left(t_{0}\right)=\bar{y}_{0}$. Then $\bar{y}_{0}+\eta_{0}$ replaces $\bar{y}_{0}$; stop or go back to 1 .

This process may be iterated until appropriate convergence tests are satisfied. (This method, incidentally, is similar to that in [3] but has the advantages that the problem solved is more general and no backwards integration is needed.)

A modification of ten helpful at step 3 is, instead, to minimize $\left\|\left[M U_{0}+N U(T)\right] \alpha+\epsilon\right\|^{2}$ under the constraints that $\left|\alpha_{j}\right| \leqq M_{j}$ where the $M_{j}$ are prescribed constants. This enables one to remain close to the current iterant $\bar{y}(0)$ in case the unconstrained solution is too large to give a useful correction.

III. The multiple-shooting method can be described as a compromise between shooting and solving implicit finite difference equations. Its only devices are a general shooting method, such as the one introduced above, and a restatement of the problem. Without loss of generality let $t_{0}=0$. The interval from 0 to $T$ is split into $k$ equal subintervals. In each subinterval, the dependent variables are denoted by new symbols. There are now $n k$ dependent variables, but the interval of integration is 0 to $T / k$. By changing the sign of $\dot{y}$ in an interval, the direction of integration in that interval is reversed. Thus, if the integration is unstable in only one direction in the original problem and the sign of $\dot{y}$ is changed in half the subintervals, then half the integrations will be in the stable direction. Moreover, the subintervals can be made short enough so that the integration will end before any instability takes over. As in finite difference methods a firm hold is kept on the solution at the splitting points. Also, all boundary conditions given in the original problem can ba satisfied at once. The new boundary conditions are that individual pieces of the same original variable must match up at the interior splitting points. Thus, if $y_{i}$ denotes a variable in the original interval from $(p-1) T / k$ to $p T / k$ and $y_{j}$ denotes the same variable in the interval from $p T / k$ to $(p+1) T / k$ and if the direction of integration in the later interval is reversed, then in general $y_{i}(0)$ and $y_{j}(0)$ are guessed and the condition introduced is that $y_{i}(T / k)=y_{j}(T / k)$. If $y_{m}$ denotes the same variable in the interval from $(p+1) T / k$ to $(p+2) T / k$, another condition is that $y_{j}(0)=y_{m}(0)$, etc.

\section{Example}

The above multiple shooting method was successful in a case in which simple shooting failed with a more accurate guess. More specifically, the integration from 0 to $T$ was sufficiently unstable to cause overflow when the initial guess was correct to three significant figures. Yet splitting the interval into four subintervals and using guesses correct to only two significant figures led to convergence. The specific problem was:

$$
\dot{y}_{1}=y_{1}^{2} / y_{2}, \quad \dot{y}_{2}=y_{2}^{2} / y_{1}, \quad y_{1}(0)=1, \quad y_{1}(4)=e^{4},
$$

with solution $y_{i}=e^{t}$.

This problem transforms into

$$
\left.\begin{array}{c}
\dot{y}_{2 i+1}=(-1) \frac{i y_{2 i+1}^{2}}{y_{2 i+2}} \\
\dot{y}_{2 i+2}=(-1) \frac{y_{2 i+2}^{2}}{y_{2 i+1}}
\end{array}\right\} \quad i=0,1,2,3,
$$

and reversing the direction of integration in the second and fourth intervals yields the boundary conditions:

$$
\begin{array}{ll}
y_{1}(0)=1 & y_{4}(0)=y_{6}(0) \\
y_{1}(1)=y_{3}(1) & y_{5}(1)=y_{7}(1) \\
y_{2}(1)=y_{4}(1) & y_{6}(1)=y_{8}(1) \\
y_{3}(0)=y_{5}(0) & y_{7}(0)=e^{4}
\end{array}
$$

A value for $y_{i}(0)$ is then guessed for $i=2,3,4,8$ (thus determining the guesses for $i=5$ and 6 ) and the general shooting procedure can be applied.

\section{REFERENCES}

1. Collatz, Lothar. The Numerical Treatment of Differential Equations. Springer, Berlin, 3d. ed., 1960.

2. Fox, L. The Numerical Solution of Two-point Boundary Problems in Ordinary Differential Equations. Oxford, 1957.

3. Goodman, T. R., and Lance, G. N. The numerical integration of two-point boundary value problems. Math. T'ables Other Aids Comp. 10 (1956), 82-86. 